\title{
TRATAMENTO DE GRANDES CISTOS RADICULARES POR MEIO DA TÉCNICA DE DESCOMPRESSÃO E POSTERIOR ENUCLEAÇÃO: RELATO DE DOIS CASOS
}

\author{
Jhonatan Thiago LACERDA-SANTOS' \\ thiagolacerda11@hotmail.com
}

\author{
Sammia Anacleto de Albuquerque PINHEIRO² \\ sammiaanacletoo@hotmail.com \\ Eduardo DIAS-RIBEIRO3 \\ eduardodonto@yahoo.com.br \\ Julliana Cariry Palhano FREIRE ${ }^{3}$ \\ jullianapalhano@hotmail.com \\ Túlio Neves de ARAÚJO ${ }^{4}$ \\ tulio_dearaujo@hotmail.com
}

\section{RESUMO}

O cisto radicular é o cisto odontogênico mais comum, podendo ser denominado de cisto radicular, cisto periapical ou cisto periodontal apical, geralmente assintomáticos e diagnosticados durante as investigações radiológicas de rotina. $O$ tratamento de cistos radiculares inclui o tratamento endodôntico não cirúrgico convencional quando a lesão é localizada, ou tratamento cirúrgico como a enucleação, marsupialização ou descompressão quando lesão é extensa. Este trabalho teve o objetivo de apresentar dois relatos de casos, que utilizaram como tratamento de cisto radicular a descompressão com posterior enucleação. Conclui-se que a técnica da descompressão como tratamento inicial dos cistos radiculares se mostrou eficaz tanto na maxila como na mandíbula, por reduzir consideravelmente o tamanho da lesão, proporcionando neoformação óssea e viabilizando a enucleação total da lesão.

\section{DESCRITORES: CISTO RADICULAR, DESCOMPRESSÃO CIRÚRGICA, TRATAMENTO CONSERVADOR.}

1 Discente do Curso de Odontologia, Faculdades Integradas de Patos (FIP), Patos-PB.

2 Professora Doutora do Curso de Odontologia, Faculdades Integradas de Patos (FIP), Patos-PB.

3 Professores do Curso de Odontologia, Unidade Acadêmica de Ciências Biológicas, Universidade Federal de Campina Grande (UFCG), campus de Patos-PB.

4 Professor do Curso de Especialização em Implantodontia, Faculdades Integradas de Patos (FIP), Patos-PB; Mestrando em Implantodontia, Faculdade São Leopoldo Mandic (SLMANDIC), Campinas-SP. Especialista em Cirurgia Buco-Maxilo-Faciais (CTBMF) e em Implantodontia, Centro Universitário de João Pessoa (UNIPÊ); João Pessoa-PB. 
Lacerda-Santos JT, Pinheiro SAA, Dias-Ribeiro E, Freire JCP, Araújo TN. Tratamento de grandes cistos radiculares por meio da técnica de descompressão e posterior enucleação: relato de dois casos. Rev. Odontol. Univ. Cid. São Paulo 2018 abr/jun 30(2) 200-9

ISSN 1983-5183

\title{
TREATMENT OF LARGE RADICULAR CYSTS BY DECOMPRESSION TECHNIQUE AND SUBSEQUENT ENUCLEATION: TWO CASE REPORTS
}

\begin{abstract}
The root cyst is the most common odontogenic cyst, and can be called root cyst, periapical cyst or apical periodontal cyst, usually asymptomatic and diagnosed during routine radiological investigations. Treatment of root cysts includes conventional non-surgical endodontic treatment when injury is localized or surgical treatment such as enucleation, marsupialization or decompression when injury is extensive. The objective of the paper has been present two case reports, which used as a treatment for root cyst decompression with subsequent enucleation. It was concluded that the decompression technique as initial treatment of the root cysts was effective both in the maxilla and in the mandible, because it considerably reduced the size of the lesion, providing bone formation and enabling the total enucleation of the lesion.
\end{abstract}

DESCRIPTORS: RADICULAR CYST - DECOMPRESSION, SURGICAL - CONSERVATIVE TREATMENT.

\section{INTRODUÇÃO}

O cisto radicular é um cisto odontogênico de origem inflamatória que surge a partir da proliferação de restos epiteliais estimulados por um processo inflamatório que se origina a partir de necrose pulpar de um dente não vital ${ }^{1,2}$. São os cistos que mais acometem a mandíbula, cerca de $52 \%$ a $68 \%$, geralmente assintomáticos e diagnosticados durante as investigações radiológicos de rotina ${ }^{1,3}$. São mais prevalentes na terceira e quarta década de vida, com maior frequência em homens ${ }^{2}$.

Uma das principais características clínicas para conclusão sobre um cisto radicular é a existência de um dente com polpa não vital, podendo apresentar tumefação e crescimento ósseo lento ${ }^{4}$. Histologicamente os cistos radiculares apresentam um revestimento epitelial escamoso estratificado, que pode demonstrar exocitose, espongiose ou hiperplasia ${ }^{5}$. Observa-se radiograficamente uma imagem radiolúcida, unilocular, redonda ou oval, com margem radiopaca bem definida na região periapical do dente acometido 3 .

O tratamento para cistos radiculares inclui o tratamento de canal não cirúrgico convencional, quando a lesão é localizada, ou tratamento cirúrgico, como enucleação, marsupialização ou descompressão, quando a lesão é grande1, 3 .

A descompressão de lesões císticas odontogênicas tem sido amplamente utilizada como um tratamento mais conservador, pois requer uma janela muito menor, criando uma abertura para a cavidade cística através da sutura de um dispositivo em sua periferia, em comparação com a marsupialização ${ }^{6}$ Na descompressão, a pequena janela mantida aberta constantemente permite drenagem e crescimento ósseo em sua periferia ${ }^{7}$, consequentemente diminuindo o tamanho da lesão cística ${ }^{8}$.

A escolha da técnica de descompressão normalmente é determinada pelo tamanho, a localização e o tipo da patologia encontrada9 . A técnica da descompressão tem sido considerada um tratamento eficaz para minimizar lesões císticas odontogênicas, por ser mais fácil de executar e mais segura para as estruturas vitais adjacentes ${ }^{7}, 10$.

O objetivo deste trabalho é relatar dois casos de cisto radicular, localizados em região posterior da mandíbula e anterior da maxila, cujo tratamento adotado foi a descompressão, seguida da enucleação total 
Lacerda-Santos JT, Pinheiro SAA, Dias-Ribeiro E, Freire JCP, Araújo TN. Tratamento de grandes cistos radiculares por meio da técnica de descompressão e posterior enucleação: relato de dois casos. Rev. Odontol. Univ. Cid. São Paulo 2018 abr/jun 30(2) 200-9

ISSN 1983-5183

da lesão.

\section{RELATO DE CASO}

\section{Caso 1}

Paciente do gênero masculino, leucoderma, 30 anos. Durante exames com finalidades ortodônticas, radiograficamente foi encontrada imagem radiolúcida, unilocular, bem delimitada, compreendendo dos elementos 34 ao 38 (Figura 1). Ao exame clínico intraoral observou-se um aumento de volume em região posterior de mandíbula, nodular, expansivo, com coloração normal, sem sinais de processo inflamatório, apresentando consistência firme à palpação e sem mobilidade.

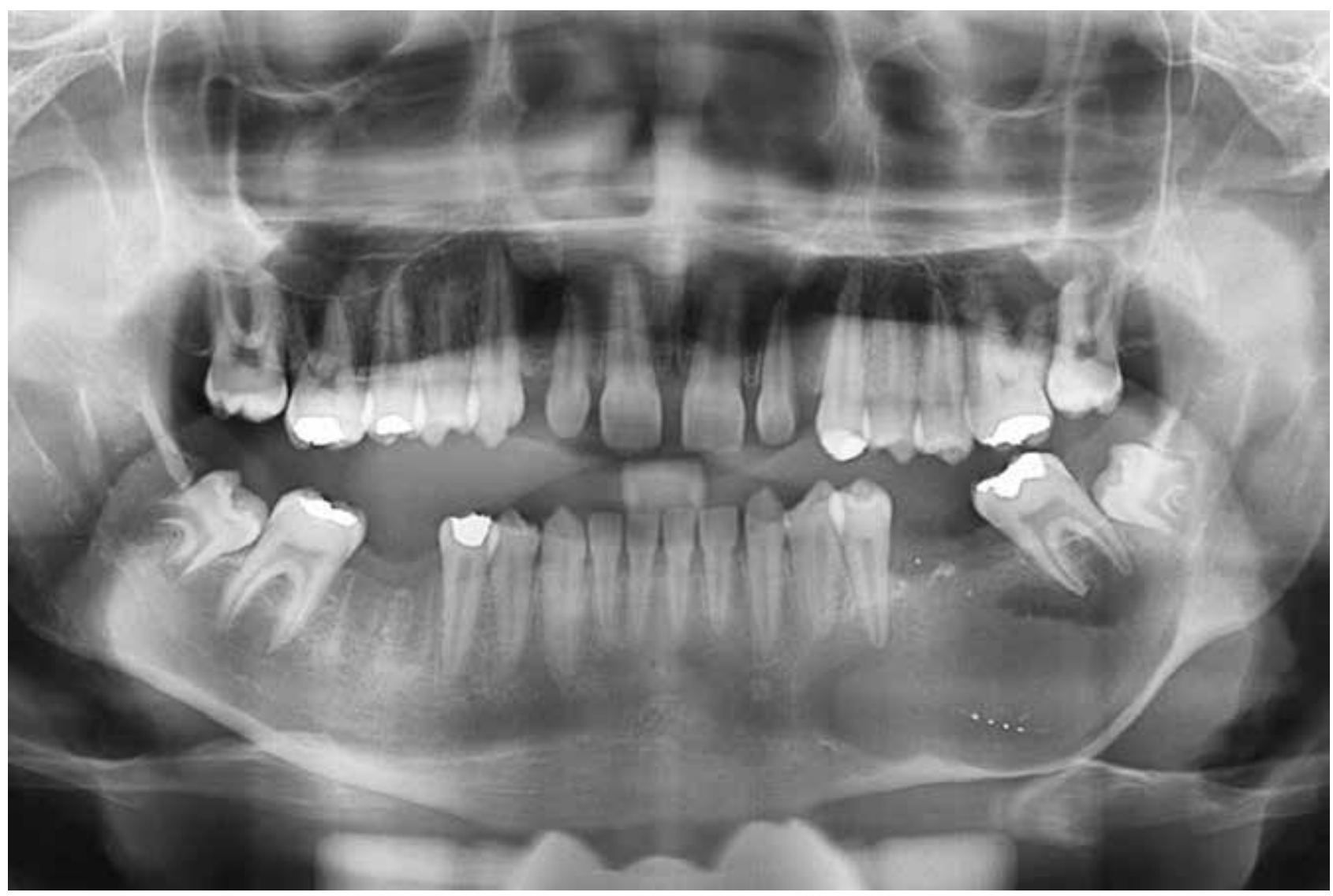

Figura 1: Radiografia panorâmica inicial.

O paciente relatou histórico de inchaço na região e há 50 dias aproximadamente realizou-se exodontia de resto radicular do elemento 36. Inicialmente foi realizada biópsia incisional, na qual, durante descolamento do retalho mucoperiosteal, observou-se reabsorção da cortical externa da mandíbula na região do elemento 36 , aproveitando-se essa janela óssea para remoção de um fragmento da membrana cística, para exame histopatológico.

Nos cortes histológicos examinados, corados em hematoxilina e eosina, observou-se fragmento de lesão cística revestida por epitélio pavimentoso estratificado não ceratinizado exibindo acantose, espongiose e exocitose. A cápsula cística é constituída por tecido conjuntivo fibroso denso, em sua maior parte, com fibras colágenas arranjadas em feixes paralelos e fibroblastos fusiformes. Intenso infiltrado 
Lacerda-Santos JT, Pinheiro SAA, Dias-Ribeiro E, Freire JCP, Araújo TN. Tratamento de grandes cistos radiculares por meio da técnica de descompressão e posterior enucleação: relato de dois casos. Rev. Odontol. Univ. Cid. São Paulo 2018 abr/jun 30(2) 200-9

ISSN 1983-5183

inflamatório com distribuição difusa, contendo linfócitos, plasmócitos e neutrófilos pode ser evidenciado. Moderada vascularização com vasos sanguíneos de tamanhos e formatos variados pode ser vista. Exsudato hemorrágico e fragmentos de tecido ósseo completam o quadro histopatológico examinado, diagnosticando assim cisto radicular.

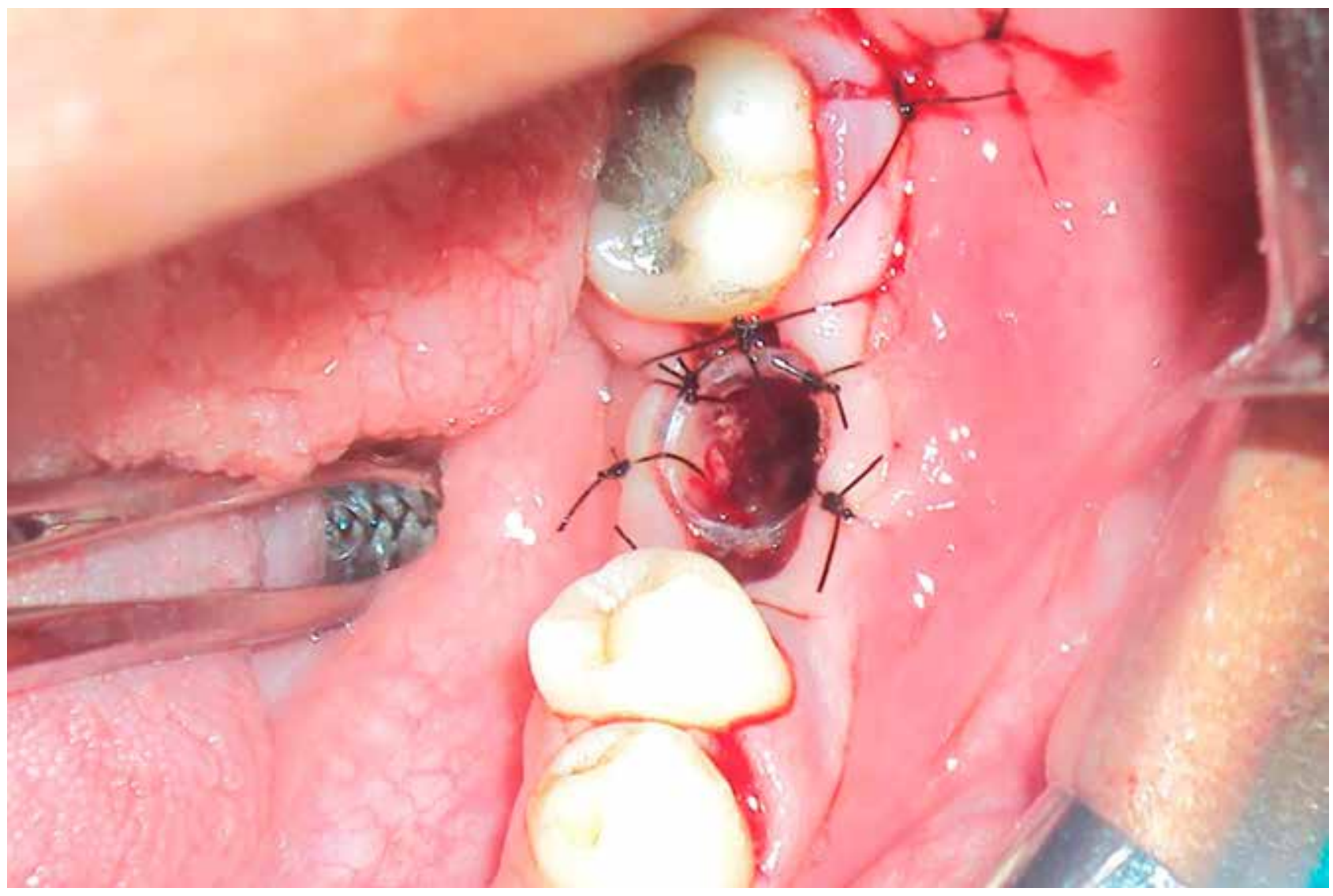

Figura 2: Instalação do dispositivo (descompressão).

Optou-se pela técnica da descompressão com enucleação, com a finalidade de diminuir a pressão interna, evitando a progressão da lesão, além de estimular a neoformação óssea. Após a instalação do dispositivo cilíndrico estéril (Figura 2), o paciente foi orientado a fazer irrigações com solução salina estéril de cloreto de sódio a $0,9 \%$, intercaladas com irrigação de digluconato de clorexidina a 0,12\% para limpeza da cavidade patológica, 3 vezes ao dia, durante o período de regressão da lesão. Após um período de 13 meses de acompanhamento através de radiografia panorâmica, observou-se a redução do cisto (Figura 3). $O$ paciente não apresentou nenhuma alteração sistêmica e foi então realizada exérese com enucleação cirúrgica total da lesão (Figura 4), sob anestesia local (Cloridrato de articaína a 4\% com epinefrina 1:100.000).

\section{Caso 2}

Paciente do gênero masculino, leucoderma, 32 anos. Destinou-se ao consultório odontológico particular para reabilitação dos elementos 11 e 21. Durante anamnese, o paciente relatou ter sido vítima de acidente doméstico há 05 anos, apresentando fratura dentária nos elementos 11 e 21.0 exame extraoral não revelou nada digno de nota, porém na avaliação intraoral observou-se abaulamento, com aumento de volume, expansivo, na região de fundo de sulco gengival anterior da maxila, apresentava consistência firme à palpação e sem mobilidade. Assim, encaminhou-se o paciente para a realização de uma tomografia 
Lacerda-Santos JT, Pinheiro SAA, Dias-Ribeiro E, Freire JCP, Araújo TN. Tratamento de grandes cistos radiculares por meio da técnica de descompressão e posterior enucleação: relato de dois casos. Rev. Odontol. Univ. Cid. São Paulo 2018 abr/jun 30(2) 200-9

\section{ISSN 1983-5183}

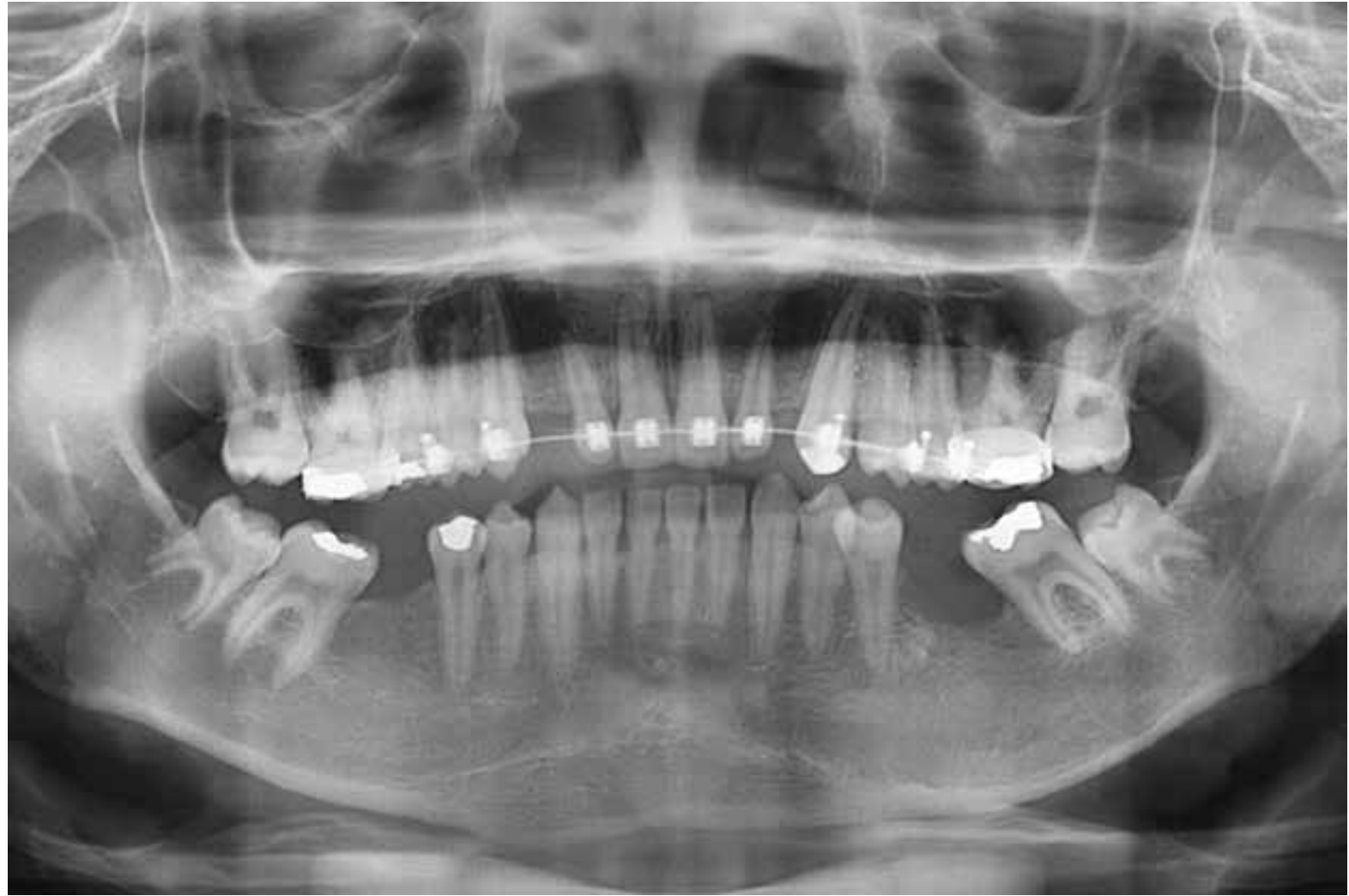

Figura 3: Radiografia panorâmica após 13 meses da descompressão.

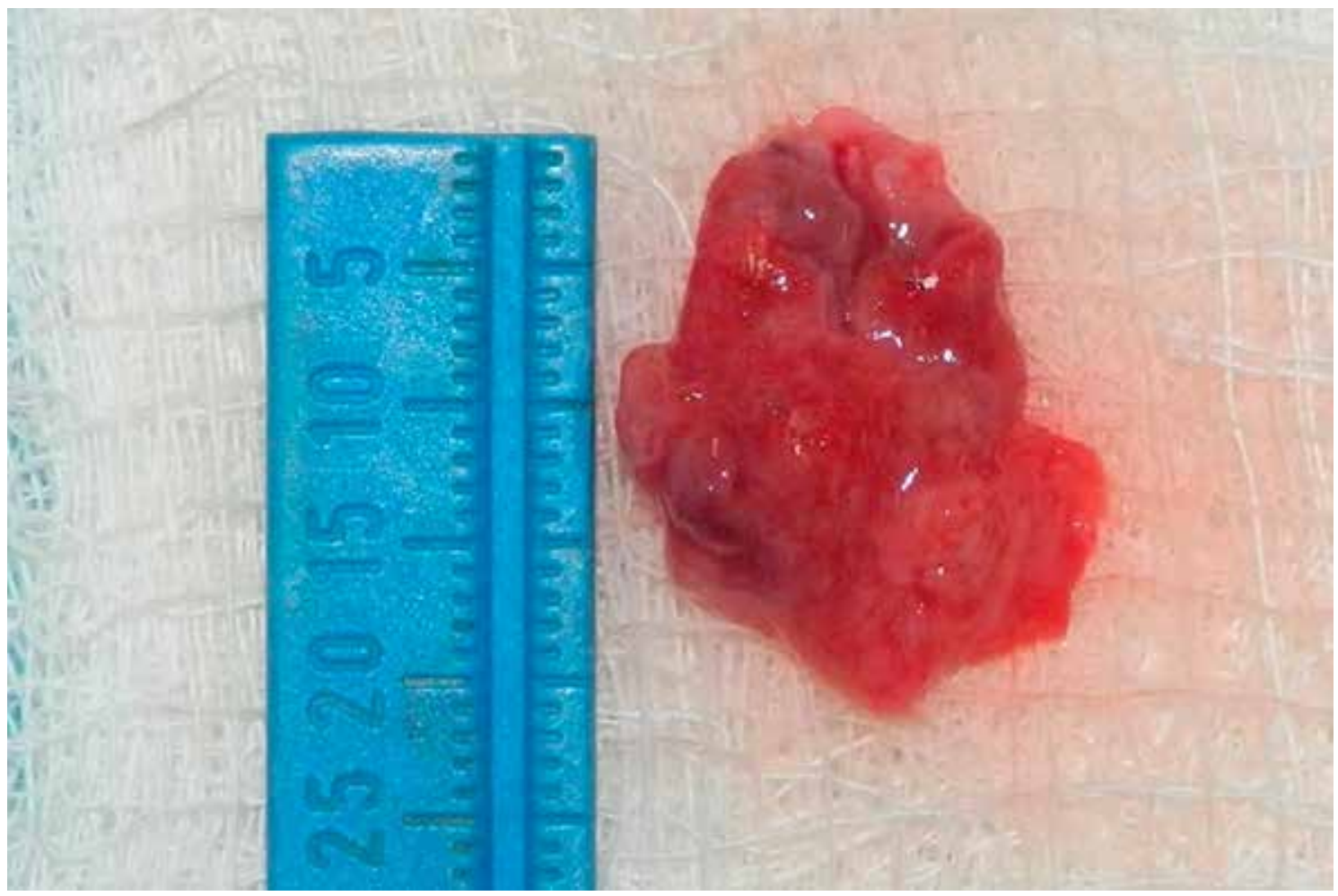

Figura 4: Peça cística após enucleação. 
Lacerda-Santos JT, Pinheiro SAA, Dias-Ribeiro E, Freire JCP, Araújo TN. Tratamento de grandes cistos radiculares por meio da técnica de descompressão e posterior enucleação: relato de dois casos. Rev. Odontol. Univ. Cid. São Paulo 2018 abr/jun 30(2) 200-9

\section{ISSN 1983-5183}

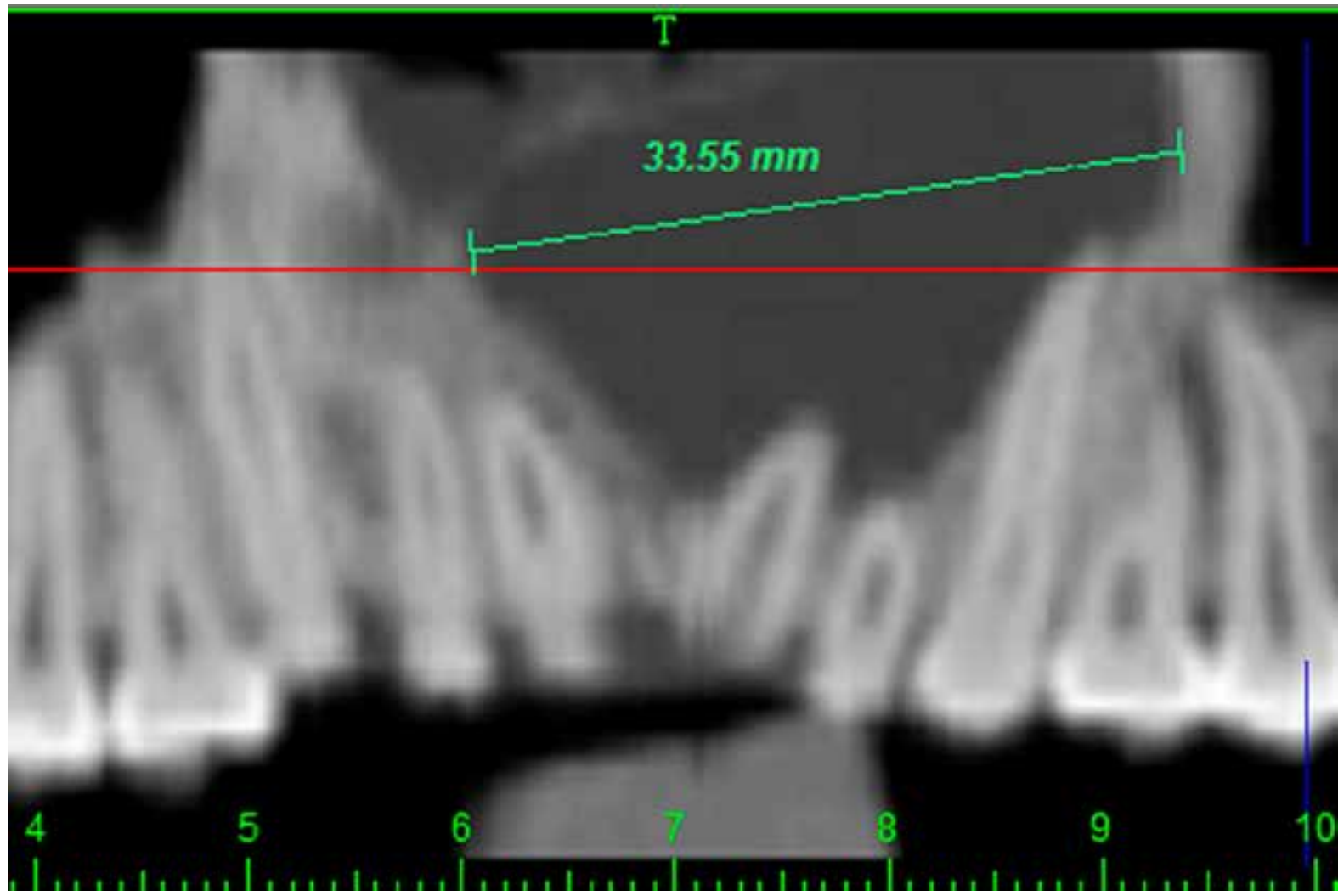

Figura 5: Corte coronal em tomografia computadorizada por feixe cônico (inicial).

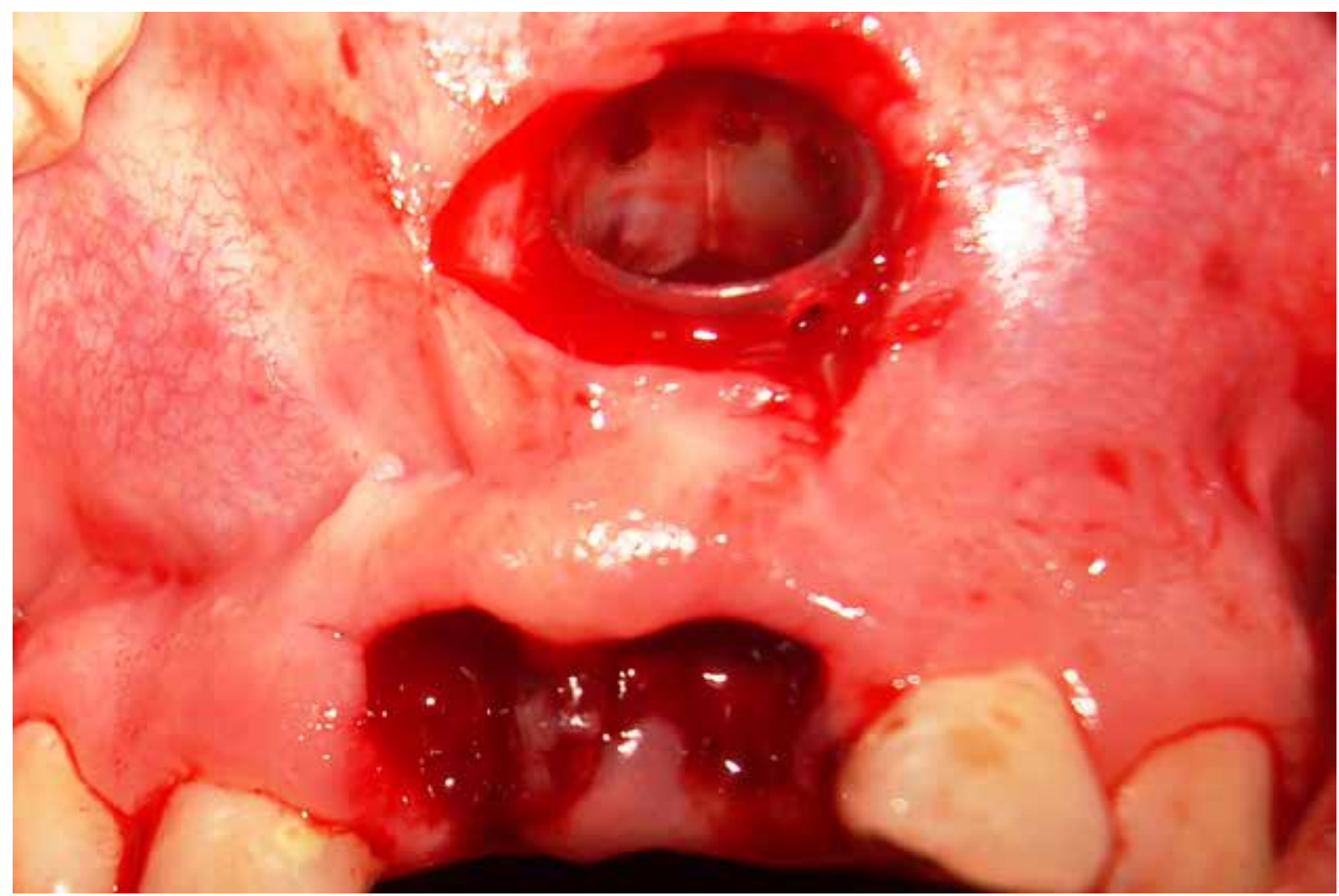

Figura 6: Instalação do dispositivo (descompressão). 
Lacerda-Santos JT, Pinheiro SAA, Dias-Ribeiro E, Freire JCP, Araújo TN. Tratamento de grandes cistos radiculares por meio da técnica de descompressão e posterior enucleação: relato de dois casos. Rev. Odontol. Univ. Cid. São Paulo 2018 abr/jun 30(2) 200-9

\section{ISSN 1983-5183}

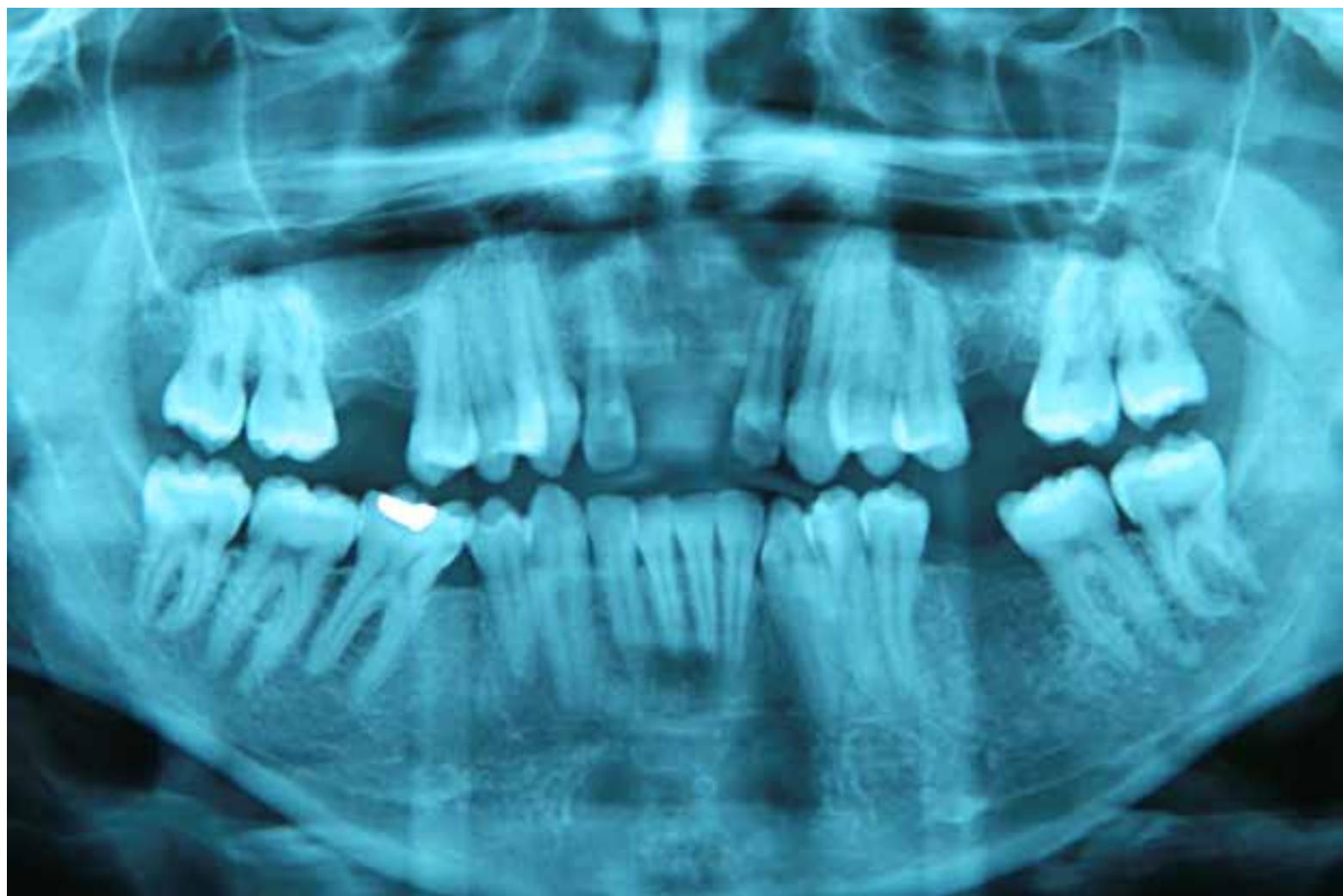

Figura 7: Radiografia panorâmica após 6 meses de descompressão.

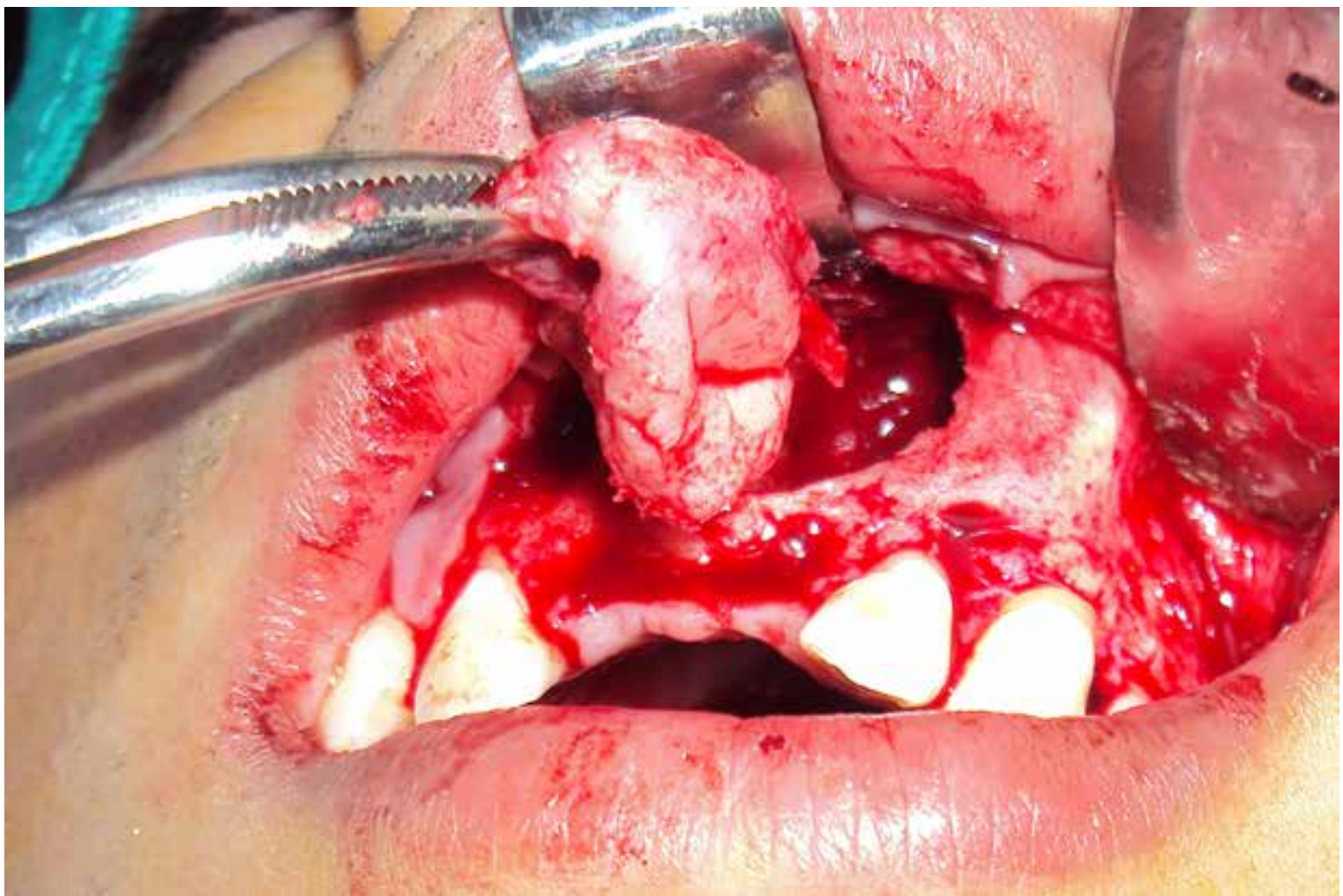

Figura 8: Enucleação total da lesão. 
Lacerda-Santos JT, Pinheiro SAA, Dias-Ribeiro E, Freire JCP, Araújo TN. Tratamento de grandes cistos radiculares por meio da técnica de descompressão e posterior enucleação: relato de dois casos. Rev. Odontol. Univ. Cid. São Paulo 2018 abr/jun 30(2) 200-9

ISSN 1983-5183

computadorizada por feixe cônico (TCFC) da região anterior da maxila para complementar o diagnóstico. 0 resultado da TCFC apresentou hiperdensidade, circunscrita, unilocular, de forma oval, com aproximadamente 33,5mm de diâmetro (Figura 5). Foi realizada punção aspirativa, biópsia incisional e encaminhamento para exame histopatológico.

Nos cortes histológicos foi diagnosticado cisto radicular. As lâminas histológicas foram coradas em hematoxilina e eosina e observou-se fragmento de lesão cística revestida por epitélio pavimentoso estratificado não ceratinizado, exibindo acantose, espongiose e exocitose. A cápsula cística era constituída por tecido conjuntivo fibroso denso, em sua maior parte, com fibras colágenas arranjadas em feixes paralelos e fibroblastos fusiformes. Intenso infiltrado inflamatório com distribuição difusa, contendo linfócitos, plasmócitos e neutrófilos. Moderada vascularização com vasos sanguíneos de tamanhos e formatos variados. Exsudato hemorrágico e fragmentos de tecido ósseo completam o quadro histopatológico.

Foi adotada a técnica da descompressão com posterior enucleação, a fim de diminuir a pressão interna, evitar a progressão da lesão, além de estimular a neoformação óssea. Após a instalação do dispositivo cilíndrico estéril (Figura 6), o paciente foi orientado a fazer irrigações com solução salina estéril de cloreto de sódio a 0,9\% intercaladas com irrigação de digluconato de clorexidina a 0,12\% para limpeza da cavidade patológica, 3 vezes ao dia, durante o período de regressão da lesão. Em um período de 6 meses de acompanhamento por meio de radiografia panorâmica, foi verificada a redução do cisto (Figura 7). 0 paciente não apresentava nenhuma comorbidade sistêmica e foi então realizada exérese com enucleação cirúrgica total da lesão (Figura 8), sob anestesia local (Cloridrato de articaína a 4\% com epinefrina 1:100.000). Depois de 2 meses, foi feito o acompanhamento radiográfico, com o qual foi possível observar a neoformação óssea.

\section{DISCUSSÃO}

O cisto radicular acomete mais indivíduos do sexo masculino $0^{2,3,5,10}$, com mais de 30 anos de idade ${ }^{2,10}$, corroborando os dados apresentados em ambos os relatos de casos. Apresenta-se com maior frequência na região anterior da maxila ${ }^{2,10}$, compatível com o segundo caso, mas também pode acometer a região posterior da mandíbula', como no primeiro caso relatado.

Uma das principais características clínicas para conclusão de um cisto radicular é a existência de um dente com polpa não vital, podendo apresentar tumefação, com crescimento ósseo lento e assintomático" 3,4. Em ambos os relatos, os cistos radiculares se apresentaram de forma assintomática, com tumefação, relacionados a um dente com polpa não vital, com grande extensão, portanto condizendo com a descrição literária.

Os cistos radiculares são caracterizados por apresentar revestimento epitelial escamoso estratificado, que pode demonstrar exocitose, espongiose ou hiperplasia ${ }^{2,5}$. As características histopatológicas mencionadas foram semelhantes aos achados nos 2 casos, onde o revestimento epitelial observado consiste em um tecido epitelial escamoso estratificado, não ceratinizado, apresentando espongiose, acantose e exocitose, cujo infiltrado inflamatório exibiu linfócitos, plasmócitos e neutrófilos.

A escolha da técnica de descompressão normalmente é determinada pelo tamanho, localização e tipo da patologia encontrada9. Por ter como característica o crescimento lento, quanto maior o cisto radicular, provavelmente maior foi seu tempo de desenvolvimento; contudo, os dois casos relatados neste artigo apresentam lesão cística de grande extensão, maiores que $20 \mathrm{~mm}$. 
Lacerda-Santos JT, Pinheiro SAA, Dias-Ribeiro E, Freire JCP, Araújo TN. Tratamento de grandes cistos radiculares por meio da técnica de descompressão e posterior enucleação: relato de dois casos. Rev. Odontol. Univ. Cid. São Paulo 2018 abr/jun 30(2) 200-9

ISSN 1983-5183

A técnica da descompressão diminui o tamanho da lesão cística e proporciona o aumento da densidade óssea, independentemente da idade do paciente ${ }^{8}$. A descompressão mostra-se mais segura em relação às estruturas vitais adjacentes ${ }^{7,10}$. Nos casos apresentados foi realizada a descompressão com posterior enucleação por serem de grande extensão e apresentarem comprometimento de estruturas anatômicas. 0 caso 1 apresentava risco de fratura patológica devido à extensão da lesão cística; com a descompressão promovida pelo tratamento, houve diminuição do cisto e consequentemente nova formação óssea, resultando numa melhor condição cirúrgica para a enucleação da lesão. Deve-se levar em consideração que as lesões císticas na mandíbula, independentemente da área onde ocorrem, têm uma taxa de redução superior comparada com a maxila ${ }^{10}$.

Não existe um parâmetro de tempo para realizar a descompressão; em geral esse tempo varia de acordo com a extensão da lesão e a idade do paciente; em pacientes com até 18 anos o tempo necessário é menor; devido a uma maior atividade osteogênica ${ }^{7}$ esse tempo pode variar de 6 a 17 meses $^{7,8,10}$. No primeiro relato deste trabalho utilizou-se a descompressão por 13 meses, enquanto que no segundo relato a descompressão foi realizada por 6 meses, e ambos resultaram em diminuição da lesão.

Para manter a janela durante a descompressão vários dispositivos podem ser utilizados, tais como: stents de resina acrílica ${ }^{8}$, sonda nasofaríngea cortada em diferentes tamanhos, embalagem de gaze iodofórmio 7 ou seringa Luer ${ }^{6}$. Alguns critérios desejáveis nesses dispositivos são: formato que impede 0 deslocamento para a cavidade cística; não interferir na mastigação e oclusão; fácil fixação com suturas, fio de aço inoxidável ou parafusos de osso; fácil instalação e remoção; superfície lisa e não porosa para impedir o acúmulo de biofilme e permitir uma limpeza fácil; confortável ao paciente; baixo custo; facilidade na irrigação da cavidade; e de fácil visualização no exame de imagem ${ }^{6}$. Nos relatos apresentados foi utilizada a técnica de irrigação preconizada pelo nosso serviço e, em ambos os casos, os pacientes foram extremamente cooperativos no que diz respeito à higiene oral e manutenção da descompressão, comparecendo a todas as avaliações pós-operatórias.

\section{CONCLUSÃO}

A técnica da descompressão como tratamento inicial dos cistos radiculares se mostrou eficaz tanto na maxila como na mandíbula, por reduzir consideravelmente o tamanho da lesão. A associação das técnicas de descompressão e enucleação de cisto radicular é um tratamento conservador eficaz que permite a preservação de estruturas nobres e evita possível fratura patológica.

\section{REFERÊNCIAS}

1. KADAM NS, Ataide Ide N, Raghava P, Fernandes M, Hede R. Management of large radicular cyst by conservative surgical approach: a case report. Journal of clinical and diagnostic research : JCDR 2014 Feb;8(2):239-41.

2. BAVA FA, Umar D, Bahseer B, Baroudi K. Bilateral radicular cyst in mandible: an unusual case report. Journal of international oral health : JIOH 2015 Feb;7(2):61-3.

3. MAHESH BS, S PS, P SM, Jyotsna TR. Role of cone beam computed tomography in evaluation of radicular cyst mimicking dentigerous cyst in a 7-year-old child: a case report and literature review. International journal of clinical pediatric dentistry 2017 Apr-Jun;10(2):213-6. 
Lacerda-Santos JT, Pinheiro SAA, Dias-Ribeiro E, Freire JCP, Araújo TN. Tratamento de grandes cistos radiculares por meio da técnica de descompressão e posterior enucleação: relato de dois casos. Rev. Odontol. Univ. Cid. São Paulo 2018 abr/jun 30(2) 200-9

ISSN 1983-5183

4. GAYNOR WN. Bilateral radicular cysts of mandibular deciduous teeth: a case report. The New Zealand dental journal 2012 Sep;108(3):106-9.

5. ULOOPI KS, Shivaji RU, Vinay C, Shrutha SP, Chandrasekhar R. Conservative management of large radicular cysts associated with non-vital primary teeth: a case series and literature review. Journal of the Indian Society of Pedodontics and Preventive Dentistry 2015 Jan-Mar;33(1):53-6.

6. CATUNDA IS, Catunda RB, Vasconcelos BC, De Oliveira HF. Decompression device for cavitary bone lesions using Luer syringe. Journal of oral and maxillofacial surgery : official journal of the American Association of Oral and Maxillofacial Surgeons 2013 Apr;71(4):7235.

7. ANAVI Y, Gal G, Miron H, Calderon S, Allon DM. Decompression of odontogenic cystic lesions: clinical long-term study of 73 cases. Oral surgery, oral medicine, oral pathology, oral radiology, and endodontics 2011 Aug;112(2):164-9.

8. GAO L, Wang XL, Li SM, Liu CY, Chen C, Li JW, et al. Decompression as a treatment for odontogenic cystic lesions of the jaw. Journal of oral and maxillofacial surgery : official journal of the American Association of Oral and Maxillofacial Surgeons 2014 Feb;72(2):32733.

9. KOLOKYTHAS A, Schlieve T, Miloro M. Simple method for securing a decompression tube for odontogenic cysts and tumors: a technical note. Journal of oral and maxillofacial surgery: official journal of the American Association of Oral and Maxillofacial Surgeons 2011 Sep;69(9):2392-5.

10. OLIVEROS-LOPEZ L, Fernandez-Olavarria A, Torres-Lagares D, Serrera-Figallo MA, Castillo-Oyague $\mathrm{R}$, Segura-Egea JJ, et al. Reduction rate by decompression as a treatment of odontogenic cysts. Medicina oral, patologia oral y cirugia bucal 2017 Sep 1;22(5):e643-e50.

RECEBIDO EM 09/11/2017

ACEITO EM 04/04/2018 